\title{
Validation of the International Tumor Budding Consensus Conference (ITBCC) 2016 recommendation in squamous cell carcinoma of the lung-a single-center analysis of 354 cases
}

\author{
Christina Neppl $^{1} \cdot$ Inti $_{\text {Zlobec }}{ }^{1} \cdot$ Ralph A. Schmid $^{2} \cdot$ Sabina Berezowska $\mathbb{D}^{1}$
}

Received: 19 July 2019 / Revised: 25 October 2019 / Accepted: 28 October 2019 / Published online: 3 December 2019

(c) The Author(s), under exclusive licence to United States \& Canadian Academy of Pathology 2019

\begin{abstract}
There are no universally accepted grading systems in pulmonary squamous cell carcinoma (pSQCC). Recently, tumor budding, cell nest size, and spread through airspaces (STAS) have been proposed as grading scheme candidates. Tumor budding is a well-established independent prognostic factor in colorectal cancer. The International Tumor Budding Consensus Conference (ITBCC) provided consensus on scoring in 2016, albeit for colorectal cancers. Here, we aimed to validate the ITBCC method in pSQCC and evaluate additional proposed grading parameters. We analyzed a fully clinico-pathologically annotated Western single-center cohort of 354 consecutive primary resected pSQCC (resected 2000-2013). Patients with SQCC of other organs were excluded to reliably exclude lung metastases. We assessed conventional grading, keratinization, STAS, and tumor budding according to ITBCC recommendations, and correlated them with clinico-pathological parameters and survival. Tumor budding was low $\left(0-4\right.$ buds $\left./ 0.785 \mathrm{~mm}^{2}\right)$ in $41 \%$, intermediate $\left(5-9\right.$ buds $\left./ 0.785 \mathrm{~mm}^{2}\right)$ in $30 \%$, and high $\left(\geq 10\right.$ buds $\left./ 0.785 \mathrm{~mm}^{2}\right)$ in $29 \%$ of cases (mean bud count $\left.=7.45(\mathrm{H} \& \mathrm{E}), \min =0, \max =84\right)$. Cell nests of $1,2-4,5-15,>15$ cells were present in $68 \%, 20 \%, 5 \%, 7 \%$, respectively. We detected STAS in $33 \%$ of cases, desmoplasia in $68 \%$. Tumor budding assessed as continuous and categorized variables was highly concordant between hematoxylin and eosin (H\&E) and pancytokeratin $($ AE1/AE3) stained slides $(P<0.001)$ and significantly associated with tumor size, UICC/AJCC pT, pN, stage (all $P<0.001$ ) and presence of mediastinal lymph node metastases (H\&E: $P=0.028$ ). Tumor budding was a significant prognostic parameter for overall, disease-specific, and progression-free survival (PFS) (all $P<0.001$ ). ITBCC tumor budding categories were independent prognostic factors for overall survival ( $\mathrm{HR}=1.581 ; 95 \% \mathrm{CI} 1.186-2.108 ; P=0.002)$, diseasespecific survival $(\mathrm{HR}=1.710 ; 95 \% \mathrm{CI} 1.111-2.632 ; P=0.015)$, and PFS $(\mathrm{HR}=1.457 ; 95 \%$ CI $1.123-1.890 ; P=0.005)$. STAS or conventional tumor grade had no prognostic value. In conclusion, we confirm tumor budding as an independent prognostic marker in pSQCC and validate the ITBCC 2016 scoring recommendations in pSQCC.
\end{abstract}

This study was presented at the 30th European Congress of Pathology in Bilbao.

Supplementary information The online version of this article (https:// doi.org/10.1038/s41379-019-0413-7) contains supplementary material, which is available to authorized users.

Sabina Berezowska

sabina.berezowska@pathology.unibe.ch

1 Institute of Pathology, University of Bern, Murtenstrasse 31, 3010 Bern, Switzerland

2 Division of General Thoracic Surgery, Inselspital University Hospital Bern, Bern, Switzerland

\section{Introduction}

Pulmonary squamous cell carcinoma (pSQCC) represents about one third of non-small cell lung cancers. There is still no recommended grading system for $\mathrm{pSQCC}$ that allows sound prognostic stratification [1].

Although there is no universally agreed on WHOrecommended grading scheme for pulmonary adenocarcinomas, prognostication is currently mostly performed according to the morphologic features of predominant growth patterns as proposed by the International Association for the Study of Lung Cancer [1, 2]. Additional morphological parameters have been evaluated for their prognostic impact, namely spread through airspaces (STAS) [3, 4], proliferative index [5], subtypes of acinar adenocarcinoma [6], and tumor budding [7, 8]. 
In pSQCC, in particular tumor budding and cell nest size including single cell invasion are considered possible candidates for grading purposes [9, 10].

Budding emerged first as a prognostic marker in colorectal cancer, where it is discussed for many decades now [11-15]. Its prognostic relevance has subsequently been established in other cancer entities such as pancreatic cancer [16, 17], oral squamous cell carcinoma [18], and cervical squamous cell carcinoma [19]. Numerous studies have been published using various approaches to count tumor buds, with differences in staining method, number of evaluated high-power fields, or categorical versus continuous values [20, 21]. Eventually, the International Tumor Budding Consensus Conference (ITBCC) 2016 provided a consensus on how to evaluate tumor budding in colorectal cancer, scoring one hotspot region in hematoxylin and eosin $(\mathrm{H} \& \mathrm{E})$ stained slides, using $\times 20$ objective magnification, thus evaluating $0.785 \mathrm{~mm}^{2}$ [22] This approach has been validated in colorectal cancer [23-25], pancreatic cancer [26], intrahepatic cholangiocarcinoma [27], cutaneous squamous cell carcinoma [28], and oral tongue squamous cell carcinoma [29]. In colorectal cancer, tumor budding is now included as an additional prognostic factor in the 8th edition of the Union for International Cancer Control's TNM classification and as a feature for reporting in the current College of American Pathologists (CAP) reporting guidelines [12, 30, 31].

Here, we aimed to validate the ITBCC scoring method in a large and well-characterized cohort of pSQCC and evaluate the additional morphological features recommended for prognostication.

\section{Materials and methods}

\section{Patient cohort and clinical parameters}

In this retrospective, single-center study, we analyzed 354 patients with primary resected pSQCC diagnosed consecutively at the Institute of Pathology, University of Bern between January 1, 2000 and December 31, 2013. The patients were naïve to preoperative treatment and have not been diagnosed with squamous cell carcinoma of other sites previously or concomitantly in order to reliably exclude metastatic pulmonary lesions. In 75 patients adjuvant chemotherapy was applied according to standard oncological guidelines. Clinical information and histological typing were reevaluated as reported previously [32].

Correct tumor typing was confirmed according to the current WHO guidelines for this study, by re-evaluating all slides using H\&E-morphology and standard immunohistochemistry with antibodies to TTF-1 and p40 in unclear cases [1]. All tumors were restaged according to the UICC 2017 TNM 8th edition [30, 33]. The details regarding the patient cohort and tumor characteristics are provided in Table 1 and as Supplementary material (Supplementary Table S1).

Overall survival (OS) was defined as the time from surgical resection to death of the patient. Time from surgical resection until disease-specific death was defined as diseasespecific survival (DSS), with censoring of patients who died due to unrelated diseases. PFS marked the time span from surgical resection to development of recurrence or death to any cause.

The study was conducted and is reported according to the REMARK-guidelines [34] and was approved by the local Ethics Commission (KEK 200/14), which waived the requirement for written informed consent.

\section{Assessment of tumor grade and desmoplasia}

For this study, all cases were graded according to the recommendations published in the cancer grading manual as follows: tumors were assigned grade 1 (well-differentiated squamous cell carcinoma) in case of prominent keratinization, easily visible intercellular bridges, and polygonal tumor cells with prominent cell borders. Tumors showing scattered foci of keratinization, less frequent intercellular bridges, smaller sized tumor cells with well-defined cell membranes, or central comedo-like necrosis were assigned grade 2 (moderately differentiated squamous cell carcinoma). Tumors were assigned grade 3 (poorly differentiated) if intercellular bridges were rare or missing, no keratin pearls formation but single cell keratinization was observed or tumors displayed sheet-like growth or single cell infiltration with prominent cytological atypia. Grades 1-3 are exemplified in the Supplementary Fig. S1. Accordingly, grade 1 and 2 of this grading scheme correspond to the current WHO classification category of keratinizing carcinomas, and grade 3 carcinomas represent non-keratinizing carcinomas [1].

Desmoplasia was noted to be present or absent.

\section{Assessment of tumor budding}

Tumor budding was assessed by a pathologist according to the scoring system proposed by the ITBCC 2016 [22]. Tumor buds were defined as tumor cell clusters of four or less tumor cells infiltrating the adjacent parenchyma. Assessment of buds was performed on H\&E stained slides. All available tumor slides per case were scanned at medium power $[\times 10]$, and slides with the highest amount of budding at the invasive front ("hotspot") were selected. Tumor buds located in one "hotspot" were counted at $\times 20$ magnification (adjusted to an area of $0.785 \mathrm{~mm}^{2}$, Nikon Eclipse $\mathrm{Ci}$ microscope; Nikon AG Instruments, Egg, Switzerland). Budding was recorded using a three-tier system as recommended (0-4 buds: low budding, BD1; 5-9 buds: 
Table 1 Clinical and pathological characteristics of the cohort in relation with tumor budding

\begin{tabular}{|c|c|c|c|c|c|c|c|c|c|}
\hline \multirow[t]{2}{*}{ Factors } & \multirow[t]{2}{*}{$n=354$} & \multicolumn{4}{|c|}{ Budding categories } & \multicolumn{4}{|c|}{ Budding continuous } \\
\hline & & $\begin{array}{l}\text { BD1 } \\
n=146(41 \%)\end{array}$ & $\begin{array}{l}\text { BD2 } \\
n=106(30 \%)\end{array}$ & $\begin{array}{l}\text { BD3 } \\
n=102(29 \%)\end{array}$ & $P$ value & H\&E mean & $P$ value & CK mean & $P$ value \\
\hline \multicolumn{10}{|l|}{ Gender } \\
\hline Male & $302(85 \%)$ & $121(34 \%)$ & $91(26 \%)$ & $90(25 \%)$ & 0.494 & 7.6 & 0.2205 & 8.5 & 0.2344 \\
\hline Female & $52(15 \%)$ & $25(7 \%)$ & $15(4 \%)$ & $12(3 \%)$ & & 7.6 & & 6.3 & \\
\hline Age, median (min-max) & $\begin{array}{l}69.09 \text { (median; } \\
43-85 \text { years) }\end{array}$ & $69.72\left({ }^{b}\right)$ & $67.81\left({ }^{b}\right)$ & $68.15\left(^{b}\right)$ & $0.086\left({ }^{a}\right)$ & $R=-0.021$ & 0.689 & $R=0.013$ & 0.8 \\
\hline $\begin{array}{l}\text { Tumor size (median; } \\
\text { min-max) }\end{array}$ & $\begin{array}{l}44.5 \mathrm{~mm} \text { (median; } \\
8-150 \mathrm{~mm})\end{array}$ & $39.91\left(^{b}\right)$ & $51.12\left({ }^{b}\right)$ & $53.67\left({ }^{b}\right)$ & $<0.001\left(^{(\mathrm{a})}\right.$ & $R=0.2$ & 0.0002 & $R=0.24$ & $<0.001$ \\
\hline \multicolumn{10}{|c|}{ pT-stage UICC TNM 8th edition } \\
\hline pT1a & $6(2 \%)$ & $5(1 \%)$ & $1(<1 \%)$ & 0 & $<0.001$ & 2.48 & $<0.001$ & 2.07 & $<0.001$ \\
\hline pT1b & $26(7 \%)$ & $12(3 \%)$ & $7(2 \%)$ & $7(2 \%)$ & & 6.26 & & 5.82 & \\
\hline pT1c & $48(14 \%)$ & $31(9 \%)$ & $7(2 \%)$ & $10(3 \%)$ & & 4.98 & & 5.46 & \\
\hline pT2a & $69(20 \%)$ & $29(8 \%)$ & $22(6 \%)$ & $18(5 \%)$ & & 6.89 & & 7.69 & \\
\hline $\mathrm{pT} 2 \mathrm{~b}$ & $52(15 \%)$ & $23(7 \%)$ & $20(6 \%)$ & $9(3 \%)$ & & 6.55 & & 7.60 & \\
\hline pT3 & $81(23 \%)$ & $33(9 \%)$ & $25(7 \%)$ & $23(7 \%)$ & & 7.87 & & 8.74 & \\
\hline pT4 & $72(20 \%)$ & $13(4 \%)$ & $24(7 \%)$ & $35(10 \%)$ & & 10.64 & & 11.90 & \\
\hline \multicolumn{10}{|c|}{ pN-stage UICC TNM 8th edition } \\
\hline pNO & $204(58 \%)$ & $104(29 \%)$ & $55(16 \%)$ & $45(18 \%)$ & $<0.001$ & 6.34 & $<0.001$ & 6.94 & $<0.001$ \\
\hline pN1 & $110(31 \%)$ & $33(9 \%)$ & $37(11 \%)$ & $40(11 \%)$ & & 8.86 & & 9.83 & \\
\hline $\mathrm{pN} 2$ & $40(11 \%)$ & $9(3 \%)$ & $14(4 \%)$ & $17(5 \%)$ & & 9.19 & & 10.48 & \\
\hline \multicolumn{10}{|l|}{ Distant metastasis (pM) } \\
\hline Absent (M0) & $344(97 \%)$ & $144(41 \%)$ & $103(29 \%)$ & $97(27 \%)$ & 0.255 & 7.36 & 0.180 & 8.05 & 0.024 \\
\hline Present (M1) & $10(3 \%)$ & $2(1 \%)$ & $3(1 \%)$ & $5(1 \%)$ & & 10.5 & & 14.88 & \\
\hline \multicolumn{10}{|c|}{ Stage UICC TNM 8th edition } \\
\hline IA1 & $4(1 \%)$ & $3(\%)$ & $1(<1 \%)$ & 0 & $<0.001$ & 2.89 & $<0.001$ & 2.48 & $<0.001$ \\
\hline IA2 & $21(6 \%)$ & $12(3 \%)$ & $4(1 \%)$ & $5(1 \%)$ & & 5.23 & & 4.76 & \\
\hline IA3 & $34(10 \%)$ & $24(7 \%)$ & $6(12 \%)$ & $4(1 \%)$ & & 3.48 & & 3.96 & \\
\hline IB & $49(14 \%)$ & $22(6 \%)$ & $17(5 \%)$ & $10(3 \%)$ & & 6.38 & & 7.0 & \\
\hline IIA & $29(8 \%)$ & $14(4 \%)$ & $11(3 \%)$ & $4(1 \%)$ & & 6.73 & & 7.44 & \\
\hline IIB & $90(25 \%)$ & $46(13 \%)$ & $21(6 \%)$ & $23(7 \%)$ & & 6.51 & & 7.59 & \\
\hline IIIA & $90(25 \%)$ & $16(5 \%)$ & $35(10 \%)$ & $39(11 \%)$ & & 10.62 & & 11.05 & \\
\hline IIIB & $27(8 \%)$ & $7(2 \%)$ & $8(2 \%)$ & $12(3 \%)$ & & 8.97 & & 10.62 & \\
\hline IVA & $7(2 \%)$ & $2(1 \%)$ & $2(1 \%)$ & $3(1 \%)$ & & 7.67 & & 12.99 & \\
\hline IVB & $3(1 \%)$ & 0 & $1(<1 \%)$ & $2(1 \%)$ & & 17.08 & & 19.28 & \\
\hline \multicolumn{10}{|l|}{ Grade } \\
\hline G1 & $7(2 \%)$ & $3(1 \%)$ & $1(<1 \%)$ & $3(1 \%)$ & 0.598 & 6.02 & 0.712 & 6.49 & 0.245 \\
\hline G2 & $176(50 \%)$ & $74(21 \%)$ & $57(16 \%)$ & $45(13 \%)$ & & 6.84 & & 7.19 & \\
\hline G3 & $171(48 \%)$ & $69(20 \%)$ & $48(14 \%)$ & $54(15 \%)$ & & 8.13 & & 9.39 & \\
\hline \multicolumn{10}{|l|}{ Pleural invasion } \\
\hline PL0 & $260(73 \%)$ & $120(34 \%)$ & $71(20 \%)$ & $69(20 \%)$ & 0.005 & 6.98 & 0.020 & 7.58 & 0.009 \\
\hline PL1 & $43(12 \%)$ & $12(3 \%)$ & $20(6 \%)$ & $11(3 \%)$ & & 7.86 & & 9.86 & \\
\hline PL2 & $28(8 \%)$ & $10(3 \%)$ & $9(3 \%)$ & $9(3 \%)$ & & 7.64 & & 8.83 & \\
\hline PL3 & $23(7 \%)$ & $4(1 \%)$ & $6(2 \%)$ & $13(4 \%)$ & & 11.64 & & 11.93 & \\
\hline \multicolumn{10}{|l|}{ Resection status } \\
\hline R0 & $306(86 \%)$ & $139(39 \%)$ & $88(25 \%)$ & $79(22 \%)$ & $<0.001$ & 6.87 & $<0.001$ & 7.61 & $<0.001$ \\
\hline $\mathrm{R} 1 / 2$ & $48(14 \%)$ & $7(2 \%)$ & $18(5 \%)$ & $23(7 \%)$ & & 11.14 & & 12.24 & \\
\hline Total & 354 & & & & & & & & \\
\hline
\end{tabular}

${ }^{\mathrm{a}}$ Kruskal-Wallis-test

${ }^{\mathrm{b}}$ Mean

intermediate budding, BD2; $\geq 10$ buds: high budding, BD3) in addition to the absolute bud counts [22]. Areas with extensive peritumoral inflammation and subsequent tumor tissue fragmentation were excluded from evaluation. To evaluate the necessity of immunohistochemical pancytokeratin (AE1/AE3) staining in the assessment of tumor budding, tumor budding was scored on AE1/ AE3 stained slides containing budding "hotspot" areas in an analogous manner in all cases. Representative BD1-BD3 cases are shown in Fig. 1a-f. 
Fig. 1 Different categories of tumor budding, cell nest size and spread through airspaces. Tumor budding category low (BD1; a, d; case \#174), intermediate (BD2; b, e; case \#172), and high (BD3; c, f; case \#343). Different categories of cell nest size, with cell nests $>15$ cells $(\mathbf{g}$; case \#162), and cell nests $5-15$ cells (h; case \#126). Exemplary case depicting spread through airspaces (i; case \#271). (BD1-BD3: a-c: HE upper panel, d-f: pancytokeratin AE1/ AE3 lower panel, overall magnification $\times 200$; cell nest size: $\mathbf{g}, \mathbf{h}$ : HE, overall magnification $\times 200$; spread through airspaces: i: HE, overall magnification $\times 50$ )
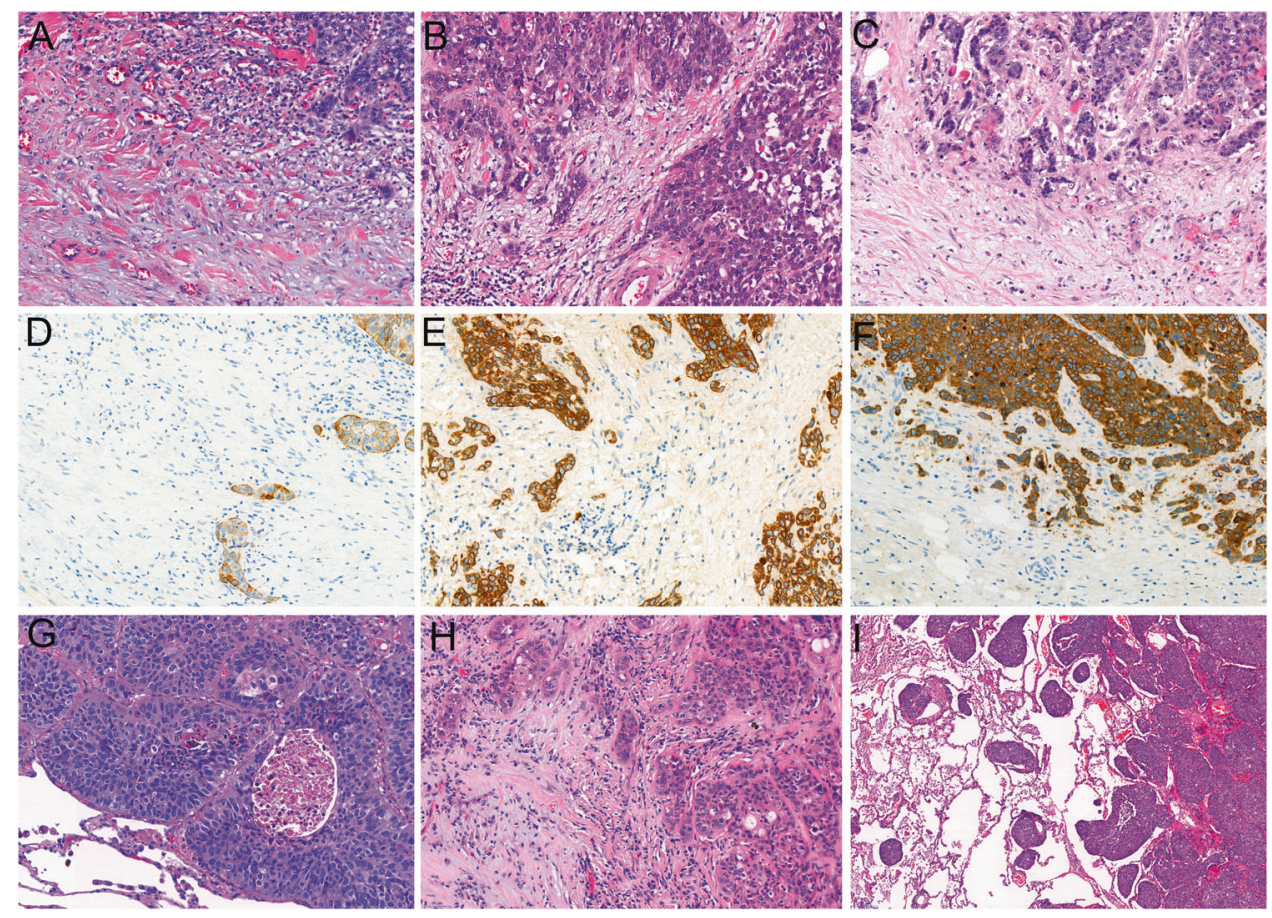

\section{Assessment of tumor cell nests}

The presence of tumor cell nests was evaluated on $\mathrm{H} \& \mathrm{E}$ stained whole slide sections as described previously $[9,10]$. Accordingly, tumor nest size was classified as single cell invasion, small tumor nests ( $2-4$ cells), intermediate tumor nests ( $5-15$ cells), and large tumor nests ( $\geq 15$ cells). Tumor nest size was evaluated at the tumor invasive front and tumor center separately, and the smallest nest size was recorded. Single cell invasion and small tumor nests correspond to tumor buds. Intermediate and large tumor nests are shown in Fig. 1g, h.

\section{Assessment of STAS}

STAS was assessed as described by Kadota and colleagues, defining STAS as spreading of tumor cell nests in airspaces beyond the edge of the main tumor (Fig. 1i) [3, 35]. Presence or absence of STAS was noted.

\section{Immunohistochemical analysis}

Whole slide sections were immunohistochemically stained on an automated immunostainer (Leica BOND RX, Leica Biosystems, Switzerland) using the following antibodies: anti-cytokeratin AE1/AE3 (Dako-Agilent, M3515 mouse monoclonal antibody; dilution 1:200, pretreatment with citrate buffer, $20 \mathrm{~min}$ at $100^{\circ} \mathrm{C}$ ); anti-TTF- 1 clone $8 \mathrm{G} 7 \mathrm{G} 3 / 1$ (Cell Marque, Rocklin, CA, USA; dilution 1:400, antigen retrieval by Tris-EDTA, $\mathrm{pH} \mathrm{9,30} \mathrm{min} \mathrm{at} 95^{\circ} \mathrm{C}$ ) and anti- p40 (Biocare Medical, Biosystems Switzerland AG, Switzerland; dilution 1:100, antigen retrieval Tris-EDTA, $\mathrm{pH} 9$, $30 \mathrm{~min}$ at $100^{\circ} \mathrm{C}$ ).

\section{Statistical analysis}

Statistical analysis was performed using IBM SPSS Statistics 24 software (IBM Corporation, Armonk, USA). Crosstabs and $\chi^{2}$-test were applied for group comparisons, and Kruskal-Wallis test or Pearson's correlation for continuous values. Agreement of bud counts assessed using H\&E and AE1/AE3 was determined using interclass correlation analysis and kappa analysis. Univariate analysis was calculated using log-rank tests and Kaplan-Meier curves, and multivariate analysis was performed using Cox regression analysis. Effect size was determined by Hazard ratios (HR) and $95 \%$ CI. $P$ values were two-sided and $P<0.05$ was considered statistically significant.

\section{Results}

\section{High correlation of tumor bud counts between H\&E and pancytokeratin stains}

Tumor budding ranged from 0 to 84 buds $/ 0.785 \mathrm{~mm}^{2}$, with a mean of 7.45 buds (H\&E; AE1/AE3: 8.24 buds). Low-grade peritumoral budding $\left(0-4\right.$ buds $\left./ 0.785 \mathrm{~mm}^{2}, \mathrm{BD} 1\right)$ was found in $41 \%$ of patients (H\&E, $n=146$; AE1/AE3: $37 \%, n=$ 


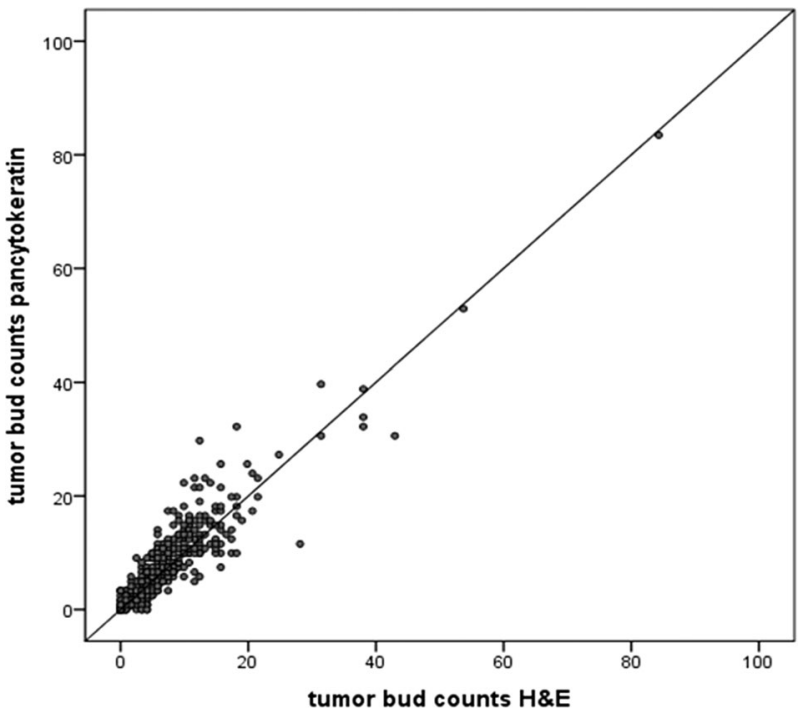

Fig. 2 Correlation between absolute tumor bud counts evaluated using H\&E and pancytokeratin AE1/AE3 stained slides (continuous values; $n=354) . R=0.92, P<0.001$

131), intermediate-grade budding (5-9 buds $/ 0.785 \mathrm{~mm}^{2}$, BD2) in 30\% (H\&E, $n=106$; AE1/AE3: 27\%, $n=96)$, and high-grade budding ( $\geq 10$ buds $\left./ 0.785 \mathrm{~mm}^{2}, \mathrm{BD} 3\right)$ in $29 \%$ (H\&E, $n=102$; AE1/AE3: $36 \%, n=126$ ).

There was a highly significant correlation of tumor bud counts at the tumor's invasive front using $\mathrm{H} \& \mathrm{E}$ and pancytokeratin AE1/AE3 stained slides, both for continuous values (Fig. 2; $r=0.92 ; P=<0.001$ ) and tumor budding categories (Table $2 ; P<0.001$ ). In addition, interclass correlation analysis confirmed a very high agreement between continuous values of tumor budding assessed using $\mathrm{H} \& \mathrm{E}$ versus AE1/AE3 (ICC $=0.91$ ). The weighted kappa coefficient showed a good level of agreement for tumor budding categories $(\mathrm{kappa}=0.78$, Table 2).

\section{Tumor budding is associated with higher tumor stage including presence of mediastinal lymph node metastases}

ITBCC assessed tumor budding showed a significant correlation with pleural invasion (H\&E: $P=0.005$; AE1/AE3: $P=0.037$, higher tumor size, higher pT-category, $\mathrm{pN}$ category, UICC/AJCC-stage (Fig. 3) and resection status (all $P<0.001, \mathrm{H} \& \mathrm{E}$ and AE1/AE3), but not with distant metastases (H\&E: $P=0.255$; AE1/AE3: $P=0.152$ ) or conventional tumor grading (H\&E: $P=0.598$; AE1/AE3: $P=0.645$ ) (Table 1). In particular, tumor budding was significantly correlated with the presence of mediastinal lymph node metastases, defining the pN2-category [36] (H\&E: $P=0.028$; AE1/AE3: $P=0.001$ ).
Cell nest sizes are associated with higher tumor stage but not the presence of mediastinal lymph node metastases

Cell nests at the invasive front were present as single cell invasion in $68 \%(n=242), 2-4$ cells/nest in $20 \%(n=71)$, $5-15$ cells/nest in $5 \%(n=17)$, and clusters of $\geq 15$ cells in $7 \% \quad(n=24)$, respectively. When analyzing the central tumoral area, $59 \%$ cases showed single cell invasion $(n=$ 207), $28 \%$ cell nests of $2-4$ cells $(n=99), 6 \%$ tumor cell nests of 5-15 cells $(n=21)$, and $8 \%$ had central cell nests of $\geq 15$ cells $(n=27)$. There was a significant correlation of cell nest size at the invasive front with cell nest size in the central tumoral area $(P<0.001)$.

The presence of smaller cell nests at the tumor invasive front significantly correlated with larger tumor size $(P=$ $0.001)$, higher pT $(P=0.028)$ and UICC-stage $(P<0.001$; Fig. 3), but not with lymph node metastases, distant metastases or conventional tumor grading (Supplementary Table S2). Cell nest size at the invasive front did not show a significant association with the presence of mediastinal lymph node metastases $(P=0.173)$.

\section{Desmoplasia is associated with tumor budding categories and cell nest size}

Desmoplasia was present in 240 cases (68\%). The presence of desmoplasia was significantly associated with higher tumor budding categories and lower cell nest size at the invasive front $(P<0.001)$. In line with this, desmoplasia was significantly associated with higher pT-stage $(P=0.001)$, larger tumor size, pN-stage, UICC-stage $(P<0.001$ each), and resection status $(P=0.002)$ (Supplementary Table S3).

STAS is associated with higher tumor stage but not the presence of mediastinal lymph node metastases

STAS was present in 115 cases (33\%). STAS was significantly associated with larger tumor size $(P=0.002)$, higher pT $(P=0.011), \mathrm{pN}(P=0.001)$, and UICC-Stage $(P=0.004)$, but not with distant metastases or tumor grade (Supplementary Table S3). STAS did not show a significant association with the presence of mediastinal lymph node metastases $(P=0.472)$.

\section{Grading shows no association with tumor size or tumor stage}

Half of the tumors were grade $2(176 / 354 ; 50 \%)$, the other half grade $3(171 / 354 ; 48 \%)$. Only $7 / 354$ (2\%) tumors classified as grade 1 .

Grading was significantly associated with resection status $(P=0.021)$, but not with other clinico-pathological 
Table 2 Agreement of tumor budding categories (H\&E and AE1/AE3) and kappa statistic (k). $P<0.001$

\begin{tabular}{llllll}
\hline & \multicolumn{2}{l}{ Pancytokeratin AE1/AE3 } & K (simple) & K (weighted) \\
\cline { 2 - 4 } H\&E & Low (BD1) & Intermediate (BD2) & High (BD3) & & \\
\hline Low (BD1) & 89 & 17 & 0 & & \\
Intermediate (BD2) & 8 & 44 & 21 & $0.69(0.62-0.77)$ & $0.78(0.72-0.84)$ \\
High (BD3) & 0 & 4 & 65 & & \\
\hline
\end{tabular}

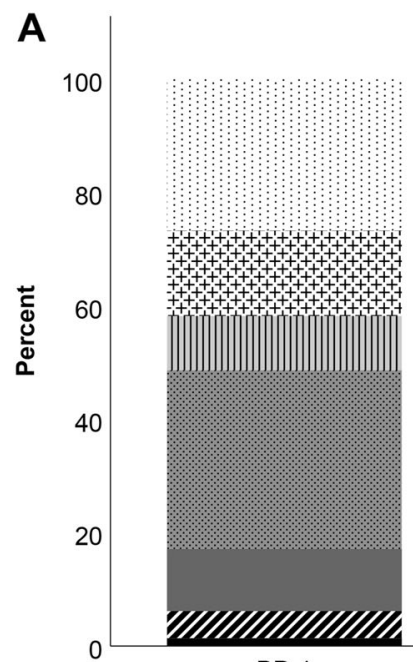

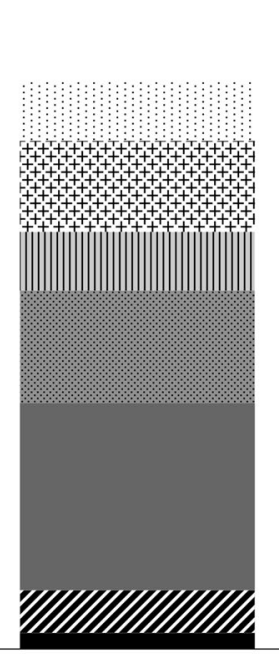

BD 2

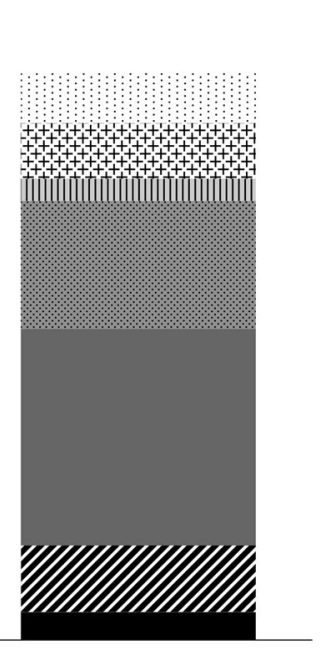

BD 3
ITBCC tumor budding

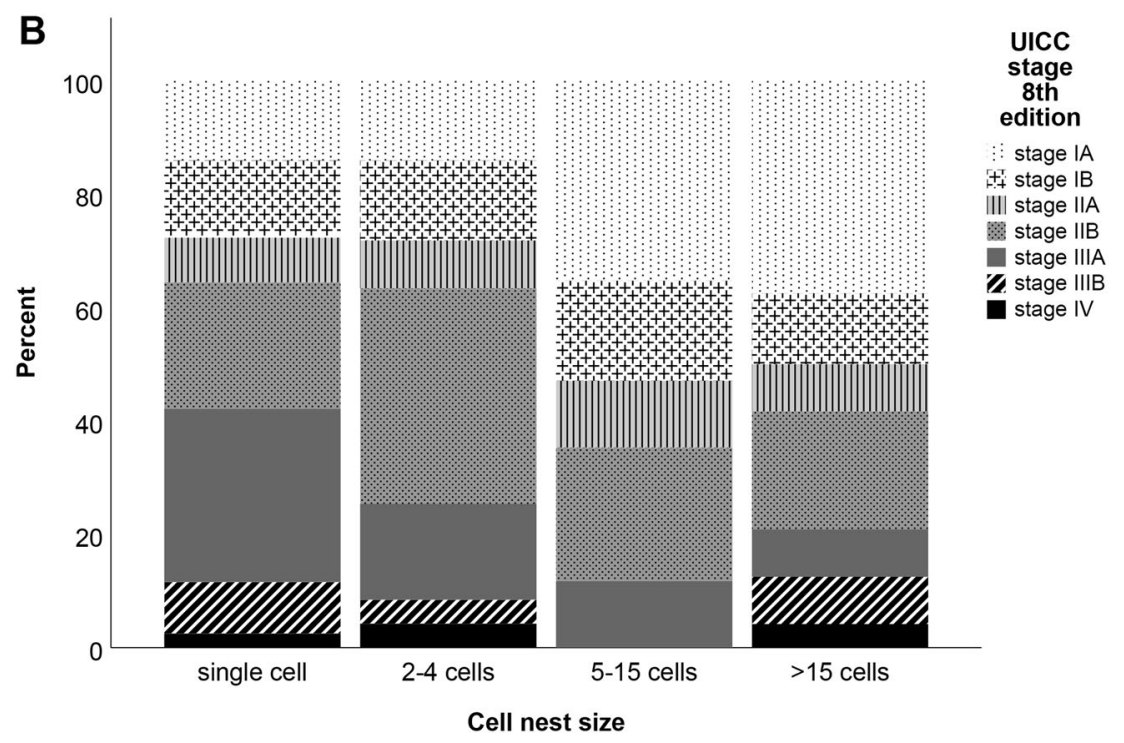

\section{UICC} stage edition

: stage IA

+. stage IB

III stage IIA

䁌 stage IIB

stage IIIA

stage IV
2. stage IIIB cell nest size categories (b) relative to tumor stage, as also presented in Table 1

Fig. 3 Bar-graphs show the distribution of cases stratified
the three different tumor according to the International Tumor Budding Consensus Conference (ITBCC) 

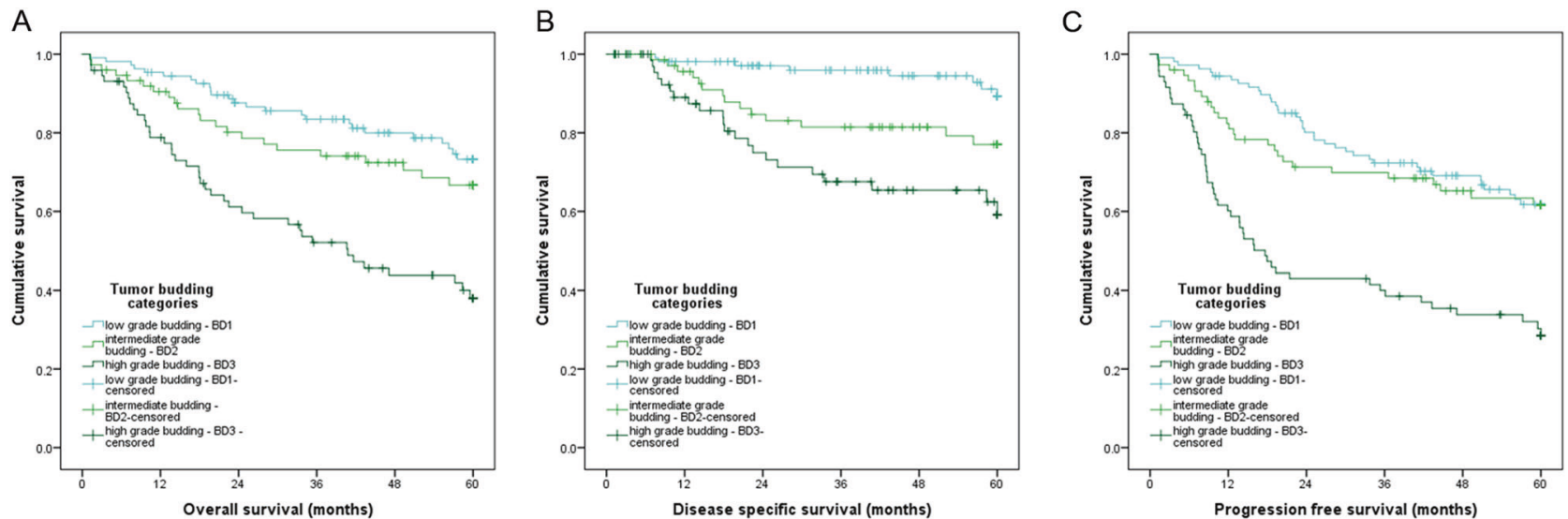

Fig. 4 Kaplan-Meier curves showing overall survival (a), diseasespecific survival (b) and progression-free survival (c) stratified by tumor budding categories assessed according to the recommendations by the International Tumor Budding Consensus Conference (ITBCC)

Adjuvant chemotherapy showed no significant association with survival (OS: $P=0.641$; DSS: $P=0.402$; PFS: $P=0.601)$.

Among the parameters assessed for this study, higher tumor budding was significantly associated with shorter OS, shorter DSS and PFS, both in ITBCC categories $(P<0.001$, all; H\&E and AE1/AE3; Fig. 4) and continuous tumor bud numbers (H\&E; OS: HR 1.039; 95\% CI: 1.022-1.055; $P<$ 0.001; DSS: HR 1.036; 95\% CI: 1.011-1.062; $P=0.004$; PFS: HR 1.037; 95\% CI 1.023-1.051; $P<0.001)(\mathrm{AE} 1 /$ AE3; OS: HR 1.047; 95\% CI: 1.032-1.062; $P<0.001$; DSS: HR 1.046; 95\% CI; 1.023-1.069; $P<0.001$; PFS: HR 1.044; 95\% CI: 1.031-1.057; $P<0.001)$.

Smaller cell nest sizes at the tumor invasive front were associated with shorter survival (OS: $P=0.022$; DSS: $P=$ 0.023; PFS: $P=0.004$; Supplementary Fig. S2).

Presence of desmoplasia was associated with shorter DSS $(P=0.013)$ and PFS $(P=0.002)$, but not OS $(P=$ 0.050).

STAS or conventional tumor grade were not associated with survival in our cohort (STAS: OS: $P=0.838$; DSS: $P=0.317$; PFS: $P=0.695$; tumor grade: $\mathrm{OS}: P=0.630$; DSS: $P=0.652$; PFS: $P=0.984$; Supplementary Fig. S3 and S4).

\section{Tumor budding is an independent prognostic marker for shorter survival}

In multivariate analysis, ITBCC tumor budding categories assessed in H\&E stained slides were an independent prognostic factor for OS, additionally to tumor size, age, and sex, as well as DSS, additionally to tumor size, and PFS, additionally to UICC-stage and age (Table 3 ).

The assessment of ITBCC tumor budding categories assessed in pancytokeratin AE1/AE3 stained slides rendered
2016 in H\&E stained slides (low budding, BD1; intermediate budding, BD2; high budding, BD3). Comparisons were conducted using a logrank test

equal results, with budding categories being an independent prognostic marker for OS, together with patient age, sex, and tumor size, as well as DSS, in addition to tumor size, and PFS, together with UICC-stage and patient age (Supplementary Table S4).

Continuous tumor bud values were an independent prognostic marker for OS and PFS, but not for DSS (Supplementary Table S5).

\section{Discussion}

Tumor grading should quantify the probable clinical aggressiveness of a neoplasm. Currently, there is no universally accepted grading scheme for pSQCC. Tumor budding at the invasion front, cell nest size, keratinization, and stromal content/desmoplasia were proposed as candidates to be used alone or in combination to grade pSQCC, due their prognostic value observed in several studies $[9,10]$.

From this list, tumor budding is a particularly promising parameter, as its association with tumor aggressiveness has been established in several tumor entities, amongst them lung cancer. In pSQCC, tumor budding consistently has been shown to be prognostic by several groups using different scoring criteria: selecting the maximal score in $1 \mathrm{HPF}$ at $\times 200$ total magnification after scanning 10 HPF [37], scoring $10 \mathrm{HPF}$ at $\times 200$ total magnification in hotspot areas [9] or $10 \mathrm{HPF}$ at $\times 400$ total magnification corresponding to $2.4 \mathrm{~mm}^{2}$ [10].

Unfortunately, the lack of consensus regarding the scoring of budding in pSQCC impedes easy implementation of this parameter into internationally accepted grading or reporting systems. In colorectal cancer, where tumor budding is established now as a prognostic marker for the 
Table 3 Multivariate analysis of tumor budding using the tumor budding scoring system proposed by the International Tumor Budding Consensus Conference (ITBCC) 2016

\begin{tabular}{|c|c|c|c|c|}
\hline \multirow[t]{2}{*}{ Factor } & \multirow[t]{2}{*}{$P$ value } & \multirow[t]{2}{*}{ HR } & \multicolumn{2}{|c|}{$95 \% \mathrm{CI}$ for $\mathrm{HR}$} \\
\hline & & & Lower & Upper \\
\hline \multicolumn{5}{|c|}{ Multivariate analysis for disease-specific survival } \\
\hline Tumor budding (ITBCC) & 0.015 & 1.710 & 1.111 & 2.632 \\
\hline UICC-stage & 0.470 & 1.105 & 0.843 & 1.448 \\
\hline Sex & 0.092 & 0.290 & 0.069 & 1.224 \\
\hline Tumor size & 0.024 & 1.018 & 1.002 & 1.033 \\
\hline Resection status & 0.099 & 1.929 & 0.884 & 4.209 \\
\hline Pleural invasion & 0.783 & 1.044 & 0.769 & 1.418 \\
\hline Desmoplasia & 0.602 & 1.246 & 0.544 & 2.855 \\
\hline \multicolumn{5}{|c|}{ Multivariate analysis for progression-free survival } \\
\hline Tumor budding (ITBCC) & 0.005 & 1.457 & 1.123 & 1.890 \\
\hline UICC-stage & 0.014 & 1.219 & 1.041 & 1.427 \\
\hline Age & $<0.001$ & 1.049 & 1.024 & 1.073 \\
\hline Sex & 0.055 & 0.521 & 0.268 & 1.013 \\
\hline Tumor size & 0.318 & 1.005 & 0.995 & 1.015 \\
\hline Resection status & 0.414 & 1.246 & 0.735 & 2.112 \\
\hline Pleural invasion & 0.685 & 1.042 & 0.853 & 1.274 \\
\hline Desmoplasia & 0.375 & 1.233 & 0.776 & 1.958 \\
\hline \multicolumn{5}{|c|}{ Multivariate analysis for overall survival } \\
\hline Tumor budding (ITBCC) & 0.002 & 1.581 & 1.186 & 2.108 \\
\hline UICC-stage & 0.192 & 1.131 & 0.940 & 1.361 \\
\hline Age & $<0.001$ & 1.059 & 1.031 & 1.089 \\
\hline Sex & 0.033 & 0.399 & 0.171 & 0.928 \\
\hline Tumor size & 0.036 & 1.012 & 1.001 & 1.023 \\
\hline Resection status & 0.089 & 1.642 & 0.927 & 2.910 \\
\hline Pleural invasion & 0.786 & 0.969 & 0.773 & 1.215 \\
\hline
\end{tabular}

longest time, scoring methodology could finally be homogenized in 2016, as a result of an international consensus conference, resulting in the ITBCC scoring method [22], enabling subsequent inclusion into the current UICC TNM classification and CAP protocol for cancer reporting $[30,31]$. Counting $10 \mathrm{HPF}$ showed no advantage over counting $1 \mathrm{HPF}$ in colorectal cancer, and is less practical in everyday practice [38].

The most practical way to go would be to extend the ITBCC scoring guidelines to other neoplasms including lung cancer, in order to avoid the confusing landscape of organ specific scoring methodology as currently experienced for e.g. determining tumor regression after neoadjuvant therapy [39]. ITBCC scoring methodology has not yet been validated in lung carcinoma.

Thus, we tested the ITBCC scoring approach on our well-characterized cohort of 354 primary resected pSQCC patients and not only confirm tumor budding as an independent prognostic factor, but also validate the ITBCC scoring scheme including the recommended 3-tier tumor budding categorization (BD1-BD3). In short, counting tumor buds in one HPF (hotspot, $\times 20$ objective magnification, $0.785 \mathrm{~mm}^{2}$ ) at the tumor invasion front as defined by the ITBCC rendered highly prognostic results and showed an association with the presence of mediastinal lymph node metastases. Of note, our cohort comprises true primary pSQCC, as patients with previous or concomitant squamous cell carcinoma of other organ systems have been excluded [32].

Addressing another controversy in discussions on tumor budding, we show that applying immunohistochemical stains for pancytokeratin does not significantly alter budding scores in pSQCC. Even though scoring budding on AE1/AE3 stained slides was subjectively easier and faster, scoring on $\mathrm{H} \& \mathrm{E}$ stained slides rendered equal results, is less costly and can be performed immediately at the time of diagnosis. AE1/AE3 staining may thus be reserved for difficult cases, without danger to overestimate the number of tumor buds. This contrasts the situation in colorectal cancer, where some cases may be underscored using $\mathrm{H} \& \mathrm{E}$ staining alone. The reason for this difference is in our experience most likely the different composition of the stroma between colorectal cancer and pSQCC. No cases in our cohort had to be excluded due to excessive inflammation or fragmentation of the tumor.

Tumor cell nest size has been proposed as an adjunct to tumor budding to be included in a new grading scheme [10]. In our cohort, smaller cell nest size was also associated with worse prognosis, though failed to be statistically significantly associated with presence of mediastinal lymph node metastases. Importantly, two of the four cell nest size increments-namely single cell invasion and small tumor nests (2-4 cells) - represent tumor buds. This renders assessment of cell nest size in routine pathological practice redundant in our view. The presence of desmoplasia was also associated with shorter survival, but likewise associated with higher budding and smaller cell nest size.

The majority of data on STAS in lung cancer focusses on adenocarcinoma, where STAS was shown to be significantly correlated with more aggressive clinicpathological features [40, 41], an increased risk of recurrence of stage I carcinomas treated by limited resection [42], and shorter survival [41]. In pSQCC, STAS has been reported to be an independent prognostic factor regarding recurrence-free survival in a Japanese cohort of 216 primary resected stage I-IV tumors [3]. STAS was also an independent factor for recurrence or cancer-specific death, but not for the OS, in a North American cohort of 445 primary resected stage I-III pSQCC [35]. In a cohort of another group from Japan, STAS was an independent prognostic factor for the recurrence and OS only in stage I, but not in higher stage pSQCC [43]. It could be speculated that the 
prognostic impact of STAS might depend on the resection procedure. STAS was shown to be an adverse prognostic factor for the OS in early stage pulmonary adenocarcinoma [44, 45] and pSQCC patients [43] treated with sublobar resection. Conversely though, other studies reported an adverse prognostic effect of STAS in adenocarcinoma patients who received lobectomy [46]. We could not validate a prognostic value of STAS in our cohort. Likewise, conventional tumor grade was not associated with survival in our cohort, again confirming the necessity to reassess the grading scheme in pSQCC.

In conclusion, we validate the independent prognostic value of tumor budding in pSQCC and ITBCC scoring methodology, thereby paving the way for harmonization of assessing tumor budding across cancer entities and organ systems.

Acknowledgements The authors gratefully acknowledge the Translational Research Unit of the Institute of Pathology for excellent technical support, and the support of the Tissue Bank Bern at the Institute of Pathology, University of Bern, in acquiring patient tissue. We are grateful to the Cancer registry Bern for support acquiring survival data.

\section{Compliance with ethical standards}

Conflict of interest The authors declare that they have no conflict of interest.

Publisher's note Springer Nature remains neutral with regard to jurisdictional claims in published maps and institutional affiliations.

\section{References}

1. Travis WD, Brambilla E, Burke AP, Marx A, Nicholson AG, editors. WHO Classification of Tumours of the Lung, Pleura, Thymus and Heart. 4th ed. Lyon: International Agency for Research on Cancer (IARC); 2015.

2. Travis WD, Brambilla E, Noguchi M, Nicholson AG, Geisinger $\mathrm{KR}$, Yatabe $\mathrm{Y}$, et al. International association for the study of lung cancer/american thoracic society/european respiratory society international multidisciplinary classification of lung adenocarcinoma. J Thorac Oncol. 2011;6:244-85.

3. Kadota K, Kushida Y, Katsuki N, Ishikawa R, Ibuki E, Motoyama $\mathrm{M}$, et al. Tumor spread through air spaces is an independent predictor of recurrence-free survival in patients with resected lung squamous cell carcinoma. Am J Surg Pathol. 2017;41:1077-86.

4. Warth A, Muley T, Kossakowski CA, Goeppert B, Schirmacher P, Dienemann $\mathrm{H}$, et al. Prognostic impact of intra-alveolar tumor spread in pulmonary adenocarcinoma. Am J Surg Pathol. 2015;39:793-801.

5. Warth A, Cortis J, Soltermann A, Meister M, Budczies J, Stenzinger A, et al. Tumour cell proliferation (Ki-67) in non-small cell lung cancer: a critical reappraisal of its prognostic role. $\mathrm{Br} \mathrm{J}$ Cancer. 2014;111:1222-9.

6. Warth A, Muley T, Kossakowski C, Stenzinger A, Schirmacher P, Dienemann $\mathrm{H}$, et al. Prognostic impact and clinicopathological correlations of the cribriform pattern in pulmonary adenocarcinoma. J Thorac Oncol. 2015;10:638-44.

7. Kadota K, Yeh YC, Villena-Vargas J, Cherkassky L, Drill EN, Sima CS, et al. Tumor budding correlates with the protumor immune microenvironment and is an independent prognostic factor for recurrence of stage i lung adenocarcinoma. Chest. 2015;148:711-21.

8. Yamaguchi Y, Ishii G, Kojima M, Yoh K, Otsuka H, Otaki Y, et al. Histopathologic features of the tumor budding in adenocarcinoma of the lung: tumor budding as an index to predict the potential aggressiveness. J Thorac Oncol. 2010;5:1361-8.

9. Kadota K, Nitadori J, Woo KM, Sima CS, Finley DJ, Rusch VW, et al. Comprehensive pathological analyses in lung squamous cell carcinoma: single cell invasion, nuclear diameter, and tumor budding are independent prognostic factors for worse outcomes. J Thorac Oncol. 2014;9:1126-39.

10. Weichert W, Kossakowski C, Harms A, Schirmacher P, Muley T, Dienemann $\mathrm{H}$, et al. Proposal of a prognostically relevant grading scheme for pulmonary squamous cell carcinoma. Eur Respir J. 2016;47:938-46.

11. Jass JR, Love SB, Northover JM. A new prognostic classification of rectal cancer. Lancet. 1987;1:1303-6.

12. Zlobec I, Lugli A. Tumour budding in colorectal cancer: molecular rationale for clinical translation. Nat Rev Cancer. 2018;18:203-4.

13. Rogers AC, Winter DC, Heeney A, Gibbons D, Lugli A, Puppa G, et al. Systematic review and meta-analysis of the impact of tumour budding in colorectal cancer. Br J Cancer. 2016;115:831-40.

14. Hase K, Shatney C, Johnson D, Trollope M, Vierra M. Prognostic value of tumor "budding" in patients with colorectal cancer. Dis Colon Rectum. 1993;36:627-35.

15. Ueno H, Murphy J, Jass JR, Mochizuki H, Talbot IC. Tumour 'budding' as an index to estimate the potential of aggressiveness in rectal cancer. Histopathology. 2002;40:127-32.

16. Karamitopoulou E, Zlobec I, Born D, Kondi-Pafiti A, Lykoudis P, Mellou A, et al. Tumour budding is a strong and independent prognostic factor in pancreatic cancer. Eur $\mathrm{J}$ Cancer. 2013;49:1032-9.

17. O'Connor KM, Li-Chang HH, Kalloger SE, Webber DL, Owen DA, Schaeffer DF. Tumor budding is an independent adverse prognostic factor in pancreatic ductal adenocarcinoma. Mod Pathol. 2014;27:452a-a.

18. Almangush A, Pirinen M, Heikkinen I, Makitie AA, Salo T, Leivo I. Tumour budding in oral squamous cell carcinoma: a metaanalysis. Br J Cancer. 2018;118:577-86.

19. Huang B, Cai J, Xu X, Guo S, Wang Z. High-grade tumor budding stratifies early-stage cervical cancer with recurrence risk. PLoS One. 2016;11:e0166311.

20. Koelzer VH, Zlobec I, Lugli A. Tumor budding in colorectal cancer-ready for diagnostic practice? Hum Pathol. 2016;47:4-19.

21. Lugli A, Karamitopoulou E, Zlobec I. Tumour budding: a promising parameter in colorectal cancer. $\mathrm{Br} \mathrm{J}$ Cancer. 2012;106:1713-7.

22. Lugli A, Kirsch R, Ajioka Y, Bosman F, Cathomas G, Dawson H, et al. Recommendations for reporting tumor budding in colorectal cancer based on the International Tumor Budding Consensus Conference (ITBCC) 2016. Mod Pathol. 2017;30:1299-311.

23. Dawson H, Galuppini F, Trager P, Berger MD, Studer P, Brugger $\mathrm{L}$, et al. Validation of the International Tumor Budding Consensus Conference 2016 recommendations on tumor budding in stage I-IV colorectal cancer. Hum Pathol. 2019;85:145-51.

24. Ueno H, Ishiguro M, Nakatani E, Ishikawa T, Uetake H, Matsuda $\mathrm{C}$, et al. Prospective multicenter study on the prognostic and predictive impact of tumor budding in stage ii colon cancer: results from the SACURA trial. J Clin Oncol. 2019;37:1886-94.

25. Landau MA, Zhu B, Akwuole FN, Pai RK. Histopathological predictors of recurrence in stage III colon cancer: reappraisal of tumor deposits and tumor budding using AJCC8 criteria. Int $\mathbf{J}$ Surg Pathol. 2019;27:147-58. 
26. Karamitopoulou E, Wartenberg M, Zlobec I, Cibin S, Worni M, Gloor B, et al. Tumour budding in pancreatic cancer revisited: validation of the ITBCC scoring system. Histopathology. 2018; 73:137-46.

27. Tanaka M, Yamauchi N, Ushiku T, Shibahara J, Hayashi A, Misumi K, et al. Tumor budding in intrahepatic cholangiocarcinoma: a predictor of postsurgery outcomes. Am J Surg Pathol. 2019;43:1180-90.

28. Fujimoto M, Yamamoto Y, Takai T, Fujimoto N, Ogawa K, Yoshikawa $\mathrm{T}$, et al. Tumor BUdding Is an Objective High-risk Factor Associated with Metastasis and Poor Clinical Prognosis in Cutaneous Squamous Cell Carcinoma Sized $<4 \mathrm{~cm}$. Am J Surg Pathol. 2019;43:975-83.

29. Xie N, Yu P, Liu H, Liu X, Hou J, Chen X, et al. Validation of the International Tumor Budding Consensus Conference (2016) recommendations in oral tongue squamous cell carcinoma. J Oral Pathol Med. 2019;48:451-8.

30. Sobin LH, Gospodarowicz MK, Christian Wittekind C, editors. International Union Against Cancer (UICC): TNM Classification of Malignant Tumours. 8th ed. Oxford, UK: Wiley-Blackwell; 2017. p. 105-12.

31. Kakar S, Shi C, Berho ME, Driman DK, Fitzgibbons P, Frankel WL, et al. Protocol for the examination of specimens from patients with primary carcinoma of the colon and rectum. College of American Pathologists (CAP). 2017. http://www.cap.org/ca ncerprotocols.

32. Keller MD, Neppl C, Irmak Y, Hall SR, Schmid RA, Langer R, et al. Adverse prognostic value of PD-L1 expression in primary resected pulmonary squamous cell carcinomas and paired mediastinal lymph node metastases. Mod Pathol. 2018;31: 101-10.

33. Neppl C, Keller MD, Scherz A, Dorn P, Schmid RA, Zlobec I, et al. Comparison of the 7th and 8th Edition of the UICC/AJCC TNM Staging System in Primary Resected Squamous Cell Carcinomas of the Lung-A Single Center Analysis of 354 Cases. Frontiers Med. 2019;6:196.

34. Altman DG, McShane LM, Sauerbrei W, Taube SE. Reporting recommendations for tumor marker prognostic studies (REMARK): explanation and elaboration. PLoS Med. 2012;9: e1001216.

35. Lu S, Tan KS, Kadota K, Eguchi T, Bains S, Rekhtman N, et al. Spread through air spaces (STAS) is an independent predictor of recurrence and lung cancer-specific death in squamous cell carcinoma. J Thorac Oncol. 2017;12:223-34.
36. Sobin LH, Gospodarowicz MK, Christian Wittekind C, editors. International Union Against Cancer (UICC): TNM classification of malignant tumours. 7th ed. Oxford, UK: Wiley-Blackwell; 2009. p. 138-46.

37. Taira T, Ishii G, Nagai K, Yoh K, Takahashi Y, Matsumura Y, et al. Characterization of the immunophenotype of the tumor budding and its prognostic implications in squamous cell carcinoma of the lung. Lung Cancer. 2012;76:423-30.

38. Rieger G, Koelzer VH, Dawson HE, Berger MD, Hadrich M, Inderbitzin $\mathrm{D}$, et al. Comprehensive assessment of tumour budding by cytokeratin staining in colorectal cancer. Histopathology. 2017;70:1044-51.

39. Langer R, Becker K. Tumor regression grading of gastrointestinal cancers after neoadjuvant therapy. Virchows Arch. 2018;472:175-86.

40. Hu SY, Hsieh MS, Hsu HH, Tsai TM, Chiang XH, Tsou KC, et al. Correlation of tumor spread through air spaces and clinicopathological characteristics in surgically resected lung adenocarcinomas. Lung Cancer. 2018;126:189-93.

41. Toyokawa G, Yamada Y, Tagawa T, Kozuma Y, Matsubara T, Haratake N, et al. Significance of spread through air spaces in resected pathological stage I lung adenocarcinoma. Ann Thorac Surg. 2018;105:1655-63.

42. Kadota K, Nitadori J, Sima CS, Ujiie H, Rizk NP, Jones DR, et al. Tumor spread through air spaces is an important pattern of invasion and impacts the frequency and location of recurrences after limited resection for small stage I lung adenocarcinomas. J Thorac Oncol. 2015;10:806-14.

43. Yanagawa N, Shiono S, Endo M, Ogata SY. Tumor spread through air spaces is a useful predictor of recurrence and prognosis in stage I lung squamous cell carcinoma, but not in stage II and III. Lung Cancer. 2018;120:14-21.

44. Shiono S, Endo M, Suzuki K, Yarimizu K, Hayasaka K, Yanagawa N. Spread through air spaces is a prognostic factor in sublobar resection of non-small cell lung cancer. Ann Thorac Surg. 2018;106:354-60.

45. Eguchi T, Kameda K, Lu S, Bott MJ, Tan KS, Montecalvo J, et al. Lobectomy is associated with better outcomes than sublobar resection in spread through air spaces (STAS)-positive T1 lung adenocarcinoma: a propensity score-matched analysis. J Thorac Oncol. 2019;14:87-98.

46. Dai C, Xie H, Su H, She Y, Zhu E, Fan Z, et al. Tumor spread through air spaces affects the recurrence and overall survival in patients with lung adenocarcinoma $>2$ to $3 \mathrm{~cm}$. J Thorac Oncol. 2017;12:1052-60. 